\title{
RELAÇÃO DOS INDICES ANTROPOMÉTRICOS E VITAMINA D COM O DESEMPENHO FUNCIONAL EM IDOSOS
}

\author{
Giselle Borges Vieira Pires de Oliveira ${ }^{1}$ \\ Fernando Lopes e Silva Júnior² \\ Maria da Conceição Barros Oliveira ${ }^{3}$ \\ Cecília Maria Resende Gonçalves de Carvalho ${ }^{4}$
}

resumo

A complexidade que abrange o processo do envelhecimento e a saúde do idoso envolve mudanças no estado nutricional, como alterações dos índices antropométricos e das concentrações de vitamina D. Este trabalho teve como objetivo analisar o desempenho funcional dos idosos e sua relação com parâmetros antropométricos e níveis de vitamina D. Trata-se de um estudo transversal e descritivo, realizado

1 Graduada em Fisioterapia pela Universidade Estadual do Piauí. Mestre em Alimentos e Nutrição da Universidade Estadual do Piauí. Fisioterapeuta vinculada ao $2^{\circ}$ Batalhão de Engenharia e Construção. E-mail: gigivieira@yahoo.com.br.

2 Graduado em Educação Física pela Universidade Federal do Piauí. Doutor em Educação Física. Professor adjunto A01 da Universidade Federal do Piauí. E-mail: flopessjunior@gmail.com.

3 Graduada em Fisioterapia pela Faculdade Mauríssio de Nassau. Doutoranda em Biotecnologia pela Universidade Federal do Piauí. E-mail: mariah.da.conceicao@hotmail.com.

4 Graduada em Nutrição pela Universidade Federal do Piauí. Doutora em Alimentos e Nutrição. Professora titular da Universidade Federal do Piaúi. E-mail: ceciliamaria.pop@hotmail.com. 
com 359 idosos assistidos por equipes da Estratégia Saúde da Família (ESF) do município de Teresina-PI, no período entre fevereiro a junho de 2011. Os idosos foram entrevistados individualmente em visitas domiciliares, utilizando-se um questionário estruturado com questões abertas e fechadas referentes aos dados sociodemográficos. Em seguida, foram submetidos ao exame bioquímico onde se realizou uma venopunção, com uma coleta de $5 \mathrm{ml}$ de sangue para posterior dosagens séricas de 250HD. Logo após, foi realizada a avaliação dos índices antropométricos, por meio de medidas de massa corporal (MC), estimativa da estatura (E), índice de massa corporal (IMC), circunferência do braço e dobra cutânea tricipital. E posteriormente, para a avaliação do desempenho funcional dos idosos foi utilizado o teste Timed Up and Go (TUG). Os resultados demonstraram que o tempo médio de realização do TUG foi 13,8s. Observou-se que massa corporal (12\%), altura (6\%), área muscular do braço (5\%) e vitamina D (29\%) foram estatisticamente significativas $(p<0,05)$ quando comparados entre os sexos. A correlação foi significativa entre associações TUG, idade ( $p=0,407, p<0,001)$ e altura $(p=-0,201, p<0,001)$. Foram encontradas correlações significativas entre o TUG, idade ( $p=0,413, p<0,001)$ e altura ( $p=-0,259$, $p<0,001)$ para o grupo feminino $(n=220)$ e correlações significativas entre o TUG, idade $(p=0,507, p<0,001)$ para o grupo masculino ( $n$ = 139). Conclui-se que dentre os parâmetros antropométricos o IMC e a circunferência abdominal foram associados com o desempenho no teste mostrando que quanto maior o IMC e CA maior o tempo de realização do teste. Os níveis séricos de Vitamina D não foram associados ao desempenho no teste.

palavras-chave

Idosos. Avaliação Funcional. Antropometria. Vitamina D.

O envelhecimento populacional é um fato incontestável reconhecido internacionalmente como uma conquista alcançada em meados do século XX e uma realidade global. A proporção de pessoas com sessenta anos ou mais está crescendo mais rapidamente que a de qualquer outra faixa etária, em todo o mundo. Entre 1970 e 2025, espera-se um crescimento de $223 \%$, ou em torno de 694 milhões, no número de idosos. Em 2025, existirá um total de aproximadamente 1,2 bilhões de pessoas com mais de sessenta anos. Até 2050 haverá dois bilhões, sendo 80\% nos países em desenvolvimento (OMS, 2014). 
O envelhecimento pode ser compreendido como um processo natural, de diminuição progressiva da reserva funcional dos indivíduos - senescência o que, em condições normais, não costuma provocar qualquer problema. No entanto, em condições de sobrecarga como, por exemplo, doenças, acidentes e estresse emocional, pode ocasionar uma condição patológica que requeira assistência - senilidade. Cabe ressaltar que certas alterações decorrentes do processo de envelhecimento podem ter seus efeitos minimizados pela assimilação de um estilo de vida mais ativo (PHILLIPS et al., 2010).

De acordo com o Censo 2010, divulgado pelo Instituto Brasileiro de Geografia e Estatística (IBGE), o Brasil apresenta dezoito milhões de pessoas com mais de sessenta anos, o que já representa $12 \%$ da população brasileira. Esses dados revelam que os idosos formam o grupo que mais cresceu na última década. Até 2025, o Brasil será o sexto país do mundo em número de idosos (IBGE, 2017). Isso se deve ao declínio da fecundidade, observada a partir dos anos 60 . O ritmo de crescimento anual do número de nascimentos passou a cair o que fez com que se iniciasse um processo contínuo de estreitamento da base da pirâmide etária, consequentemente, de envelhecimento da população (DE PAULO et al., 2012).

Esses recentes aumentos na expectativa de vida têm chamado atenção sobre as condições de saúde durante os anos adicionais de vida e sobre o impacto na morbidade, mortalidade e perdas funcionais e motoras em pessoas acima de sessenta anos. Nas alterações de saúde relacionadas com a idade, percebe-se que estão presentes fatores de risco e ocorrência de doenças crônicas não-transmissíveis que determinam ao idoso um certo grau de dependência, relacionado com a perda de autonomia e dificuldade de realizar as atividades da vida diária (MELLO et al., 2010). Dentro desse contexto, a capacidade funcional surge como um novo paradigma de saúde (CARDOSO et al., 2012).

A capacidade funcional (CF) é definida como a habilidade física e mental para manter uma vida independente e autônoma com realização plena de uma tarefa ou ação pelo indivíduo (FIEDLER; PERES, 2008). Compreende desde atividades básicas até as ações mais complexas da rotina diária. As informações geradas pela avaliação funcional possibilitam conhecer o perfil dos idosos utilizando-se ferramenta simples e útil, que pode auxiliar na definição de estratégias de promoção de saúde para os idosos, visando retardar ou prevenir as incapacidades (CAMARA et al., 2008).

Nos idosos observa-se uma diminuição da capacidade funcional, sobretudo relacionado à depreciação das funções físicas, como diminuição da função dos sistemas osteomuscular, cardiorrespiratório e nervoso, condição que prejudica os idosos a realizar suas atividades da vida diária com eficiência. 
Essa diminuição é referida como fator de aumento no risco de quedas, principalmente devido ao comprometimento na realização de tarefas do dia a dia, com limitações de força muscular, equilíbrio, marcha e mobilidade (CAMPOS, M.; VIANNA; CAMPOS, A., 2013; CARDOSO et al., 2012).

As principais alterações anatômicas e funcionais, relacionadas com o envelhecimento e ligadas ao déficit de desempenho funcional são as alterações na composição e na forma corporal, como diminuição da estatura, distribuição da gordura corporal, perda de massa muscular, diminuição da massa óssea e o declínio de aptidões psicomotoras que, em conjunto, podem provocar instabilidade postural ou incapacidade de manutenção do equilíbrio, em situações de sobrecarga funcional (GOMES; PINTO; SOAR, 2011; FELIX; SOUZA, 2009). Alterações na composição corporal e, consequentemente, no estado nutricional, também são observadas com as mudanças em que o organismo é submetido durante o envelhecimento. A inadequação nutricional também afeta o bem-estar de longevos, causando declínio funcional, devido aos aportes deficitários de calorias e nutrientes (desnutrição calórico - protéica, deficiência de vitaminas e minerais), pelo excesso calórico (sobrepeso e obesidade) ou pela utilização excessiva de substâncias como o álcool, o que leva a alterações na capacidade de realizar as atividades da vida diária (SOARES et al. 2012).

Aspectos nutricionais são importantes, pois contribuem para modulação das mudanças fisiológicas relacionadas à idade e no desenvolvimento de doenças crônicas não transmissíveis, como obesidade, osteoporose e diabetes (RUIZ et al., 2014; SILVA et al., 2008). Destaca-se a vitamina D, que tem um papel importante no aumento da força muscular e também no equilíbrio postural e dinâmico (COSTA; CARVALHO, 2012).

Diante da complexidade dos fatores relacionados à capacidade funcional de idosos, torna-se fundamental o aprofundamento desses fatores nos idosos da comunidade, visto que são escassas as pesquisas nesse grupo populacional. Portanto, o objetivo principal desse trabalho foi investigar a associação entre os índices antropométricos e níveis séricos de vitamina D com a capacidade funcional em idosos da comunidade de Teresina-PI. Hipotetizou-se que idosos com maiores valores de IMC irão apresentar menor desempenho funcional, e aqueles com maiores concentração de vitamina $\mathrm{D}$ e área muscular do braço, irão apresentar melhor desempenho motor. 
A população do estudo constituiu-se de idosos da comunidade de ambos os sexos que residiam em áreas assistidas por equipes da Estratégia Saúde da Família (ESF) do município de Teresina-PI. Dados do censo demográfico 2010 apontam 814.230 habitantes em Teresina, sendo 107.882 habitantes idosos (IBGE, 2017). A ESF possuía 66.799 idosos cadastrados na zona urbana, o que representava $89,5 \%$ de acompanhamento da população nesta faixa etária.

$\mathrm{O}$ dimensionamento amostral foi calculado utilizando-se a estimativa da prevalência de quedas de $30 \%$, com base em estudo sobre a temática na população idosa (SBGG, 2001), e erro amostral de 5\%, obtendo, consequentemente, como amostra representativa dessa população, no mínimo, 322 idosos. Considerando a possibilidade de perdas ou recusas acrescentou-se 37 idosos a essa estimativa que resultou em uma amostra de 359 idosos. Os sujeitos do estudo foram selecionados por técnica de amostragem aleatório simples (tabela de números aleatórios), com distribuição proporcional ao número de idosos cadastrados nas Coordenadorias Regionais de Saúde. Assim, foram sorteados 115 idosos na Coordenadoria Regional Centro-Norte, 117 na Leste-Sudeste e 127 na Sul.

Realizou-se o sorteio nas coordenadorias em duas etapas. A primeira consistiu da seleção de duas Unidades Básicas de Saúde (UBS) de cada Coordenadoria Regional de Saúde. A segunda fase foi o sorteio de uma equipe da ESF das unidades básicas de saúde escolhidas na primeira etapa. Em cada equipe sorteada, a lista nominal de todos os idosos que possivelmente atenderiam aos critérios de inclusão foi organizada e novo sorteio foi realizado para selecionar os idosos da pesquisa. Todo o processo de sorteio (UBS, Equipes de Saúde da Família e sujeitos do estudo) foi feito por meio da tabela de números aleatórios. Foram realizadas três tentativas de entrevistas domiciliares e, quando do terceiro retorno, não se obteve êxito na realização do inquérito, por não localização ou óbito, o caso foi considerado não resposta ou perda, sendo aceitáveis perdas de sujeitos da pesquisa que não ultrapassassem $5 \%$ da amostra total.

Para a segunda fase da pesquisa, de análise laboratorial, utilizou-se uma subamostra de cem idosos, calculada com base na estimativa do desvio padrão de 28,5ng/mL de 25(OH)D3 (SARAIVA et al., 2007). A margem de erro e o nível de confiança foram de $5,6 \mathrm{ng} / \mathrm{mL}$ e $95 \%$, respectivamente. 
Foram incluídos no estudo pessoas com idade igual ou superior a sessenta anos, que faziam parte da clientela da ESF. Foram excluídos idosos com déficit cognitivo acentuado; idosos com limitação severa de visão ou audição, não compensadas com uso de óculos ou aparelhos de amplificação sonora; e idosos que necessitassem de cadeira de rodas, condição que impossibilitaria a realização do teste motor funcional desta pesquisa. Ainda, foram excluídos os idosos que faziam suplementação de vitamina D e/ou cálcio, e aqueles com problemas renais e hepáticos crônicos para a dosagem de 25-hidroxivitamina D.

Os procedimentos e riscos pertinentes ao estudo foram explicados anteriormente à obtenção do consentimento livre e esclarecido. Os procedimentos experimentais desse estudo foram aprovados pelo Comitê de Ética para estudos com seres humanos da Universidade Federal do Piauí (Parecer CEP no $0386.0 .045 .000-10)$.

\subsection{Coleta de dados}

A coleta de dados foi realizada no período de fevereiro a junho de 2011, por meio de entrevistas individuais domiciliares, utilizando um questionário estruturado com questões abertas e fechadas. O acesso aos endereços dos idosos foi feito pelo telefone através do contato da equipe da pesquisa com as enfermeiras e os agentes comunitários de saúde das equipes sorteadas, responsáveis pelo cadastro das famílias na ESF.

Antes da coleta de dados, desenvolveu-se um estudo-piloto com 32 idosos (10\% da amostra) com características semelhantes aos sujeitos de interesse do estudo, mas que não fariam parte da amostra selecionada, como meio de verificar a adequação do instrumento de coleta de dados aos propósitos da pesquisa e padronizar o método de coleta de dados. Todos os pesquisadores de campo foram treinados para a aplicação do questionário e avaliação do teste motor funcional previamente ao estudo-piloto.

\subsection{Exame bioquímico}

Na segunda fase da pesquisa, os idosos selecionados foram contatados por telefone para agendamento do dia e horário (no turno matutino) da coleta de sangue para exames bioquímicos e orientações sobre o exame e a necessidade de jejum mínimo de oito horas. A coleta foi realizada na Unidade Básica de Saúde da ESF em que o idoso estava cadastrado ou no domicílio, caso o participante apresentasse dificuldade importante ou impossibilidade 
de deslocamento até a UBS. A venopunção foi feita por uma técnica de enfermagem contratada, respeitando-se as condições de antissepsia e segurança, segundo as Recomendações da Sociedade Brasileira de Patologia Clínica/ Medicina Laboratorial para coleta de sangue venoso (2010). Todo o processo de coleta foi realizado de acordo com as recomendações do fabricante do kit de dosagem utilizado (DiaSorin ${ }^{\circledR}$ ), sendo realizadas as dosagens séricas de 25OHD utilizando-se kits de quimioluminescência direta (DIASORIN, 2003).

\subsection{Caracterização do estado nutricional}

Realizou-se avaliação dos índices antropométricos no período da manhã, por meio de medidas de massa corporal (MC), estimativa da estatura (E), circunferência do braço, circunferência da cintura (CC) e índice de massa corporal (IMC). A massa corporal foi aferida em balança eletrônica digital portátil da marca Plena (PAÍS), com capacidade de $150 \mathrm{~kg}$. A estatura foi verificada por meio de um estadiômetro profissional portátil com escala de $1 \mathrm{~cm}$. Para avaliação da circunferência do braço utilizou-se uma fita métrica flexível. A circunferência da cintura (CC) foi mensurada com uma fita métrica metálica, com escala de $1 \mathrm{~mm}$. Em seguida, foi realizado o IMC. As avaliações antropométricas seguiram as padronizações contidas em Petroski (2007).

\subsection{Teste de desempenho funcional}

O teste de desempenho funcional utilizado foi o Timed Up and Go (TUG), este é um teste funcional simples e bastante utilizado na prática clínica. Foi validado (BOTOLFSEN et al., 2008), desenvolvido e baseado no teste Get up and go (levantar e caminhar), este tendo por finalidade avaliar o desempenho funcional. Nesse teste o paciente é solicitado a levantar-se de uma cadeira (altura do assento de $45 \mathrm{~cm}$ e dos braços de $65 \mathrm{~cm}$ ), deambular três metros, retornar e sentar-se novamente, enquanto o tempo despendido na realização desta tarefa é cronometrado. O resultado do TUG teve como parâmetro o tempo em que o participante levou para realizar o teste (PODSIADLO; RICHARDSON, 1991).

Os equipamentos necessários para a bateria de testes foram: cronômetro, cadeira de encosto reto, sem braços, com aproximadamente $45 \mathrm{~cm}$ de altura e fita de três metros de comprimento. Todas as manobras necessárias para a realização dos testes foram demonstradas inicialmente pelos avaliadores. 
A análise dos dados foi realizada com Statistical Package for Social Sciences (SPSS) para Windows (versão 19.0). Para testar a normalidade da distribuição dos dados, o teste de Kolmogorov-Smirnov foi utilizado. Teste $t$-Student para amostras independentes foi utilizado para comparar os grupos (mulheres $\mathrm{x}$ homens) nas variáveis de tempo TUG, vitamina $\mathrm{D}$, a pressão arterial sistólica, massa corporal, altura e índice de massa corporal. E o Teste $U$ de Mann-Whitney foi utilizado para comparar os grupos nas variáveis: idade, área muscular do braço e da pressão arterial diastólica. Associações entre as medidas de tempo TUG e índices antropométricos e vitamina D foram analisadas pelo Teste $\rho$ de Spearman (para o grupo total e grupo de mulheres) e teste de correlação produto momento de Pearson (grupo de homens). Valores de $\mathrm{p}<0,05$ foram considerados significativos em testes de hipóteses bicaudais. Foi realizada a modelagem múltipla com todas as variáveis que apresentaram $p<0,20$ na análise descrita no item de fatores associados ao teste Timed Up and Go. O valor do $p$ determinou a ordem de entrada no modelo múltiplo. O processo de modelagem foi o stepwise forward. A variável independente permaneceu no modelo múltiplo se $\mathrm{p}<0,05$ e/ou se for variável de ajuste.

\section{Resultados}

No presente estudo, a população foi predominantemente composta por mulheres, no grupo etário de 60 a 69 anos e que moravam acompanhados do cônjuge. A pesquisa mostrou que a média de massa corporal e estatura foi maiores no sexo masculino que no sexo feminino (Tabela 1). Constatou também que idosos do sexo masculino apresentaram maiores concentrações séricas de vitamina D quando comparados a idosos do sexo feminino, diferença estatisticamente significante. 
Tabela 1 - Características dos participantes. Valores expressos em média \pm desvio padrão.

\begin{tabular}{lcccc}
\hline & $\begin{array}{c}\text { Total } \\
(\mathrm{n}=359)\end{array}$ & $\begin{array}{c}\text { Women } \\
(\mathrm{n}=220)\end{array}$ & $\begin{array}{c}\text { Men } \\
(\mathrm{n}=139)\end{array}$ & p-value \\
Idade, anos & $70.51 \pm 8.03$ & $69.76 \pm 8.12$ & $71.69 \pm 7.78$ & 0.029 \\
Massa corporal, $\mathrm{kg}$ & $62.63 \pm 12.02$ & $59.98 \pm 11.56$ & $66.83 \pm 11.55$ & 0.001 \\
Estatura, $\mathrm{m}$ & $1.58 \pm 0.13$ & $1.55 \pm 0.14$ & $1.64 \pm 0.07$ & 0.001 \\
IMC, $\mathrm{kg} / \mathrm{m} 2$ & $25.12 \pm 5.64$ & $25.06 \pm 4.67$ & $25.22 \pm 6.91$ & 0.799 \\
CC, $\mathrm{cm}$ & $97.84 \pm 10.75$ & $98.49 \pm 10.96$ & $96.80 \pm 10.36$ & 0.074 \\
AMB, $\mathrm{cm}{ }^{2}$ & $24.45 \pm 3.64$ & $24.03 \pm 4.06$ & $25.10 \pm 2.72$ & 0.001 \\
PAS, mmHg & $131.39 \pm 22.26$ & $131.25 \pm 24.12$ & $131.63 \pm 19.05$ & 0.874 \\
PAD, mmHg & $78.12 \pm 10.91$ & $77.97 \pm 11.60$ & $78.34 \pm 9.75$ & 0.750 \\
TUG, seconds & $13.81 \pm 4.84$ & $14.01 \pm 4.95$ & $13.48 \pm 4.68$ & 0.330 \\
Vitamina D & $22.51 \pm 8.03$ & $20.12 \pm 6.12$ & $25.94 \pm 9.21$ & 0.001 \\
\hline
\end{tabular}

Notas: IMC = índice de massa corporal; $C C$ = circunferência da cintura; $\mathrm{AMB}=$ área muscular do braço; PAS = pressão arterial sistólica; PAD = pressão arterial diastólica; TUG = teste timed up and go.

Fonte: Autor da pesquisa.

O tempo médio para a realização do teste pelos idosos foi de $13,81 \pm 4.84$ segundos. O sexo está fortemente associado à ocorrência da dependência funcional, sendo mais de duas vezes maior a chance para as mulheres em relação aos homens.

Dentre as variáveis da pesquisa, observou-se correlação estatisticamente significante entre a idade e a estimativa da estatura na amostra total da presente pesquisa com o tempo de realização do TUG (Tabela 2). Idosas com excesso de peso eram mais lentas na realização do TUG. Não houve uma tendência de diferença significante entre os sexos, no presente estudo, para a variável circunferência abdominal. E não houve correlação significante entre os níveis séricos de Vitamina D e o tempo do teste desses indivíduos. 
Tabela 2 - Correlações entre o Teste Timed Up and Go (TUG) e medidas mensuradas em idosos.

\begin{tabular}{ll}
\hline Group & TUG \\
Total & \\
Idade, anos & $0.407^{*}$ \\
Massa corporal, kg & -0.069 \\
Estatura, m & $-0.201^{*}$ \\
CC, cm & 0,066 \\
IMC, $\mathrm{kg} / \mathrm{m}^{2}$ & 0.018 \\
AMB, $\mathrm{cm}^{2}$ & -0.024 \\
PAS, mmHg & -0.007 \\
PAD, mmHg & -0.023 \\
Vitamina D & -0.062
\end{tabular}

\section{Mulheres}

Idade, anos

$0.413^{*}$

Massa corporal, $\mathrm{kg}$

$-0.026$

Estatura, $m$

$-0.259^{*}$

$\mathrm{IMC}, \mathrm{kg} / \mathrm{m}^{2}$

0.072

AMB, $\mathrm{cm}^{2}$

0.033

$\mathrm{CC}, \mathrm{cm}$

0.131

PAS, $\mathrm{mmHg}$

0.019

$\mathrm{PAD}, \mathrm{mmHg}$

$-0.084$

Vitamina D

0.060

\section{Homens}

ldade, anos

$0.507^{*}$

Massa corporal, kg

$-0.092$

Estatura, $m$

$-0.071$

IMC, $\mathrm{kg} / \mathrm{m}^{2}$

$-0.087$

$\mathrm{AMB}, \mathrm{cm}^{2}$

$-0.089$

$\mathrm{CC}, \mathrm{cm}$

$-0.022$

PAS, $\mathrm{mmHg}$

$-0.080$

$\mathrm{PAD}, \mathrm{mmHg}$

$-0.019$

Vitamina D

$-0.161$

Notas: IMC = índice de massa corporal; $C C$ = circunferência da cintura; $A M B$ = área muscular do braço; PAS = pressão arterial sistólica; PAD = pressão arterial diastólica; TUG = teste timed up and go. ${ }^{*} \mathrm{p}<0.001$.

Fonte: Autor da pesquisa. 
A maior longevidade feminina em relação à masculina é um fenômeno que tem sido atribuído à menor exposição a fatores de risco, notadamente no trabalho, diferenças quanto à atitude em relação a doenças e incapacidades e, por último, maior cobertura da assistência ginecológica e obstétrica. Fato que ficou conhecido como feminização da velhice (VIRTUOSO et al., 2012).

Dados semelhantes aos encontrados na presente pesquisa foram observados em outros estudos com idosos, em que os homens apresentaram médias de estatura e massa corporal superior a das mulheres (MENEZES; MARUCCI, 2005). E quando avaliados em relação ao IMC, não houve diferença estatisticamente significante entre os sexos. Esse resultado é contrário a outros estudos nacionais e internacionais (ARROYO et al., 2007) que observaram discrepância significante nessa variável entre os sexos. Vale ressaltar que o IMC é um dos indicadores mais utilizados em estudos epidemiológicos, associado ou não a outras variáveis antropométricas, para avaliar indivíduos em risco nutricional (WHO, 2005).

Os níveis séricos de vitamina $\mathrm{D}$ diminuem com a idade, principalmente como resultado da restrição de exposição solar, diminuição da capacidade da pele em sintetizar vitamina $\mathrm{D}$ e ingestão reduzida na dieta. A deficiência de vitamina $\mathrm{D}$ em idosos está relacionada ao desenvolvimento de hiperparatireoidismo secundário e aumento do risco de fraturas (SARAIVA et al., 2007).

No que diz respeito ao desempenho funcional dos idosos encontrados nesse estudo, a média similar ao valor encontrado em um estudo com idosas na Carolina do Norte, Estados Unidos (ARNADOTTIR; MERCER, 2000). Ademais, não houve diferença significante nas comparações entre os sexos na capacidade funcional. Entretanto, médias inferiores $(9,0 \mathrm{~s}, 10,0 \mathrm{~s}, 8,3 \mathrm{~s}, 9,8 \mathrm{~s}, 8,1 \mathrm{~s}, 10,2 \mathrm{~s})$ foram encontradas em outros estudos com idosos (BISCHOFF-FERRARI et al., 2009).

Idosos que realizam o teste em um tempo menor que vinte segundos apresentam baixo risco de quedas e independência parcial, já aqueles que realizam em tempo superior a vinte segundos caracterizam alto risco de quedas e déficit de desempenho funcional (PODSIADLO; RICHARDSON, 1991). Ainda, estudos prévios mostram que o declínio da capacidade funcional está relacionado com fatores demográficos, socioeconômicos e de condições de saúde (BOYD et al., 2005; CHENG et al., 2002; ALVES et al., 2007; MACIEL; GUERRA, 2005).

Estudos sobre fatores associados à incapacidade funcional de idosos mostraram que esta esteve fortemente associada com o sexo, sendo as mulheres mais propensas a apresentar prejuízo funcional do que os homens (ALVES et 
al., 2007). Algumas hipóteses tentam explicar essa diferença, a primeira está associada à maior sobrevivência das mulheres em relação aos homens. A segunda refere-se à maior prevalência de condições incapacitantes não-fatais entre as mulheres. A terceira seria atribuída à habilidade da mulher reportar maior número de condições de saúde em relação aos homens da mesma idade (ALVES et al., 2007).

Outros estudos (SOARES et al., 2012) encontraram associação entre o estado nutricional (IMC, circunferência da panturrilha) e desempenho funcional no TUG em idosos da comunidade, assim como em nosso estudo. Quanto maior o IMC do idoso maior foi o tempo para a realização do teste.

De acordo com Ferrucci et al. (2000), em um estudo realizado com 3.381 idosos (> 71 anos), concluiu que o baixo desempenho nos testes relacionados aos membros inferiores (equilíbrio, "sentar e levantar" e velocidade de caminhar, somados em um único escore) apresentou associação significativa ( $p<0,01)$ com maior IMC, corroborando com o resultado desse estudo. Alguns fatores tentam explicar a associação entre obesidade e o baixo desempenho funcional nos testes motores (SOARES et al., 2012). Dentre eles, o excesso de gordura aumenta a sobrecarga corporal, dificultando e aumentando o estresse nas articulações e músculos, favorecendo, dessa maneira, ao maior grau de incapacidade nos idosos obesos, além de aumentar o risco de osteoartrite nos joelhos (VASCONCELOS; DIAS, J.; DIAS, R., 2007).

A medida da circunferência abdominal (CA) não é só indicador direto, mas também preditora da adiposidade abdominal e está associada à inatividade física, podendo levar à incapacidade nas atividades básicas (AVD) e instrumentais de vida diária. O acúmulo de gordura visceral aumenta a demanda energética para atividades funcionais como a deambulação. A influência da circunferência abdominal sobre o desempenho funcional de idosas, constatando que mulheres idosas com circunferência abdominal superior a $88 \mathrm{~cm}$ tiveram pior desempenho nos testes funcionais, sugerindo que a obesidade abdominal pode contribuir para o declínio funcional precoce e consequente incapacidade nessa população (BARBOSA et al., 2007).

Em um estudo sobre Vitamina D, força muscular, capacidade funcional e equilíbrio em pacientes em diálise peritoneal com deficiência de vitamina $\mathrm{D}$, constatou-se que, após a suplementação de vitamina $\mathrm{D}$, os indivíduos realizaram o TUG em menor tempo, porém nosso estudo não corrobora com este trabalho, já que houve uma fraca associação (TASKAPAN et al., 2011). Em outro estudo de revisão sistemática e de meta-análise sobre o efeito da suplementação de vitamina D na força muscular, marcha e equilíbrio de idosos, Muir e Montero-Odasso (2011), concluíram que a suplementação de 
vitamina $\mathrm{D}$ promove uma diminuição do tempo para completar o teste Timed Up and Go, mostrando efeito benéfico sobre a força e equilíbrio. Deve-se fazer suplementação de vitamina $\mathrm{D}$ em idosos com insuficiência ou deficiência para melhorar a força e desempenho funcional (ZHU et al., 2010).

A suplementação de vitamina $\mathrm{D}$ promove uma diminuição do tempo para completar o TUG, mostrando efeito benéfico sobre a força e equilíbrio. Deve-se fazer suplementação de vitamina $\mathrm{D}$ em idosos com insuficiência ou deficiência para melhorar a força e desempenho funcional (ZHU et al., 2010).

\section{Conclusão}

A identificação desses fatores é essencial para determinar, na assistência básica à saúde, métodos preventivos e de intervenção, com o objetivo de preservar ou melhorar a capacidade funcional dos idosos, prevenindo danos motores e internações. Dentre os parâmetros antropométricos o IMC e a circunferência abdominal foram associados com o desempenho no teste mostrando que quanto maior o IMC e CA maior o tempo de realização do teste. Os níveis séricos de Vitamina D não foram associados ao desempenho no teste.

Nesse contexto, sugerem-se estratégias para mudança do perfil desses fatores, pois a maioria são modificáveis, com exceção do sexo feminino e idade avançada. Orientações sobre acompanhamento médico, incentivo à prática de atividade física e hábitos saudáveis, além de orientações nutricionais que favoreçam a preservação do desempenho funcional destes idosos.

O estudo apresentou limitações devido às características transversais e correlacional do trabalho. Também não é possível estabelecer relação causal entre fatores ou determinar se índices antropométricos e níveis séricos de vitamina D investigados atuam como marcadores de subjacentes de incapacidade funcional. Entretanto, novos estudos devem ser realizados com o propósito de elucidar a correlação entre índices antropométricos e nutricionais com a capacidade funcional de idosos.

\section{Contribuições Individuais:}

Oliveira e Carvalho trabalharam na concepção do trabalho, na pesquisa, metodologia e redação final, Silva Júnior trabalhou na metodologia, análise e revisão final do artigo. 
abstract

The complexity covering the aging process and the health of the aged involves changes in nutritional status, such as changes in anthropometric indices and vitamin D concentrations. This study aimed to analyze the functional performance of the aged and its relationship with anthropometric parameters and levels of vitamin D. It is a transversal and descriptive study with 359 aged people assisted by teams of the Family Health Strategy (FHS) in the city Teresina-PI in the period from February to June 2011. The aged were interviewed individually during home visits, using a structured questionnaire with open and closed questions relating to sociodemographic data. They were then subjected to biochemical tests were conducted a venipuncture through a collection of $5 \mathrm{ml}$ of blood for subsequent serum levels of $250 H D$. Soon after, it was carried out the evaluation of anthropometric indices, using body mass ( $\mathrm{MC}$ ), height estimate $(\mathrm{E})$, body mass index (BMI), arm circumference and triceps skinfold thickness. And later, to evaluate the functional performance of the aged was used test Timed Up and Go (TUG). The results showed that the mean duration of the TUG was 13,8 s. It was observed that body weight (12\%), height (6\%), muscle area of the arm (5\%) and vitamin D (29\%) were statistically significant $(p<0.05)$ when compared between the sexes. Correlation coefficients were significant associations between TUG, age ( $p=0.407, p<0.001)$ and time $(p=-0.201, p<0.001)$. Significant correlations were found between the TUG, age ( $p=0.413$, $p<0.001)$ and height $(p=-0.259, p<0.001)$ for the female group $(n=220)$ and significant correlations between the TUG, age $(p=0.507$, $p<0.001)$ for the male group ( $n=139$ ). It is concluded that among the anthropometric parameters BMI and waist circumference were associated with performance on the test showing that the higher BMI and higher CA the test performance time. Serum levels of vitamin D were not associated with performance on the test.

keywords

Elderly. Functional Evaluation. Anthropometry. Vitamin D. 
ALVES, Luciana Correia et al. A influência das doenças crônicas na capacidade funcional dos idosos do Município de São Paulo, Brasil. Caderno de Saúde Pública, Rio de Janeiro, v. 23, n. 8, p. 1924-1930, ago. 2007.

ARNADOTTIR, Solveig; MERCER, Vicki. Effects of footwear on measurements of balance and gait in women between the ages of 65 and 93 years. Physical Therapy, Kettering, v. 80, n. 1, p. 17-27, jan. 2000.

ARROYO, Patrícia et al. Indicadores antropométricos, composición corporal y limitaciones funcionales en ancianos. Revista Médica de Chile, Santiago, v. 135, n. 7, p. 846-854, 2007.

BARBOSA, Aline Rodrigues et al. Estado Nutricional e Desempenho Motor de Idosos de São Paulo. Revista da Associação Médica Brasileira, São Paulo, v. 53, n. 1, p. 75-79, 2007.

BISCHOFF-FERRARI, Heike et al. Fall prevention with supplemental and active forms of vitamin D: a meta-analysis of randomised controlled trials. British Medical Journal, London, v. 339, n. b3692, p. 1-11, oct. 2009.

BOTOLFSEN, Pernille et al. Reliability concurrent validity of the expanded timed up and go test in older people with impaired mobility. Physiotherapy Research International, United States, v. 13, n. 2, p. 94-106, jun. 2008.

BOYD, Cynthia et al. Frailty, hospitalization, and progression of disability in a cohort of disabled older women. American Journal of Medicine, Philadelphia, v. 118, n. 11, p. 1225-1231, nov. 2005.

CAMARA, Fabiano Marques et al. Capacidade funcional do idoso: formas de avaliação e tendência. Acta Fisiatr, São Paulo, v. 15, n. 4, p. 249-256, jun. 2008.

CAMPOS, Maria Pereira Silva; VIANNA, Luciano Gabriel; CAMPOS, Amanda da Rocha. Os testes de Equilíbrio, Alcance Funcional e "Timed Up and Go" e o risco de queda em idosos. Revista Kairós Gerontologia, São Paulo, v. 16, n. 4, p. 125-138, ago. 2013.

CARDOSO, José Augusto et al. Análise da capacidade funcional dos idosos de porto alegre e sua associação com autopercepção de saúde. Estudos Interdisciplinares sobre o Envelhecimento, Porto Alegre, v. 17, n. 1, p. 111-124, fev. 2012.

CHENG, Yin et al. Self-rated economic condition and the health of elderly persons in Hong Kong. Social Science \& Medicine, Hong Kong, v. 55, n. 8, p. 1415-1424, 2002.

COSTA, Ivone Fontes Oliveira llha; CARVALHO, Carlos Mauricio Rodrigues. Vitamina D: deficiência na pessoa idosa e papel na adiposidade corporal. Revista da Sociedade Portuguesa de Ciências da Nutrição e Alimentação, Porto, v. 18, n. 1, p. 19-27, jan. 2012.

DE PAULO, Thaís Reis da Silva et al. O exercício físico funcional para idosos institucionalizados: um novo olhar para as atividades da vida diária. Estudos Interdisciplinares sobre o Envelhecimento, Porto Alegre, v. 17, n. 2, p. 413-427, mai. 2012.

DIASORIN. 25-Hydroxyvitamin D Liaison Kit Instruction Manual. Catalog n 26757-K-, Stillwater, MN, USA, 2003.

FELIX, Luciano Novaes; SOUZA, Eliane Matos. Avaliação nutricional de idosos em uma instituição por diferentes instrumentos. Revista de Nutrição de Campinas, Campinas, v. 22, n. 4 , p. 571-580, 2009.

FERRUCCI, Luigi et al. Subsystems contributing to the decline in ability to walk: bridging the gap between epidemiology and geriatric practice in the InCHIANTI study. Journal of the American Geriatrics Society, New York, 2000. 
FIEDLER, Maria Morais; PERES, Keila Gabriela. Capacidade funcional e fatores associados em idosos do Sul do Brasil: um estudo de base populacional. Cadernos de Saúde Pública, Rio de Janeiro, v. 24, n. 2, p. 409-415, fev. 2008

GOMES Ricardo Costa; PINTO Carlos Silva; SOAR Carolina. Prevalência de desnutrição em idosos institucionalizados. In: Encontro Latino Americano de Iniciação Científica e Encontro Latino Americano de Pós-Graduação, 14., 2011, São José dos Campos. Anais... São José dos Campos: Universidade do Vale do Paraíba - UNIVAP, 2011.

INSTITUTO BRASILEIRO DE GEOGRAFIA E ESTATíSTICA (IBGE). Panorama. Censo demográfico. Rio de Janeiro, 2017. Disponível em: <https://cidades.ibge.gov.br/brasil/ rj/rio-de-janeiro/panorama> Acesso em: 22 maio 2018.

MACIEL, Álvaro Campos Cavalcanti; GUERRA, Ronaldo Oliveira. Fatores associados à alteração da mobilidade em idosos residentes na comunidade. Revista Brasileira de Fisioterapia, São Paulo, v. 9, n. 1, p. 17-23, 2005.

MELLO, Renato Gorga Bandeira et al. Vitamina D e prevenção de quedas em idosos: uma revisão sistemática. Scientia Medica, Porto Alegre, v. 20, n. 2, p. 200-206, mai. 2010.

MENEZES, Tarciana Nobre de; MARUCCI, Maria de Fátima Nunes. Antropometria de idosos residentes em instituições geriátricas. Revista de Saúde Pública, São Paulo, v. 39, n. 2 , p. $169-175,2005$

MUIR, Susan; MONTERO-ODASSO, Manuel. Effect of vitamin D supplementation on muscle strenght, gait and balance in older adults: a systematic review and meta-analysis. Journal of the American Geriatrics Society, New York, 2011.

ORGANIZACIÓN MUNDIAL DE SALUD (OMS). Caídas, nota descriptiva n 344. 2014.

PETROSKI, Edio Luiz. Antropometria: técnicas e padronizações. 3. ed. Blumenau: Nova Letra, 2007.

PHILLIPS, Edward Matos et al. Interruption of Physical Activity Because of Illness in the Lifestyle Interventions and Independence for Elders Pilot Trial. Journal of Aging and Physical Activity, Birmingham, v. 18, n. 1, p. 61-74, jan. 2010.

PODSIADLO, Diane; RICHARDSON, Sandra. The Timed "Up and Go": a test of basic functional mobility for frail elderly persons. Journal of American Geriatrics Society, Philadelphia, v. 39, n. 2, p. 142-148, fev. 1991

RUIZ, Fernando Sousa et al. Associação entre deficiência de vitamina D, adiposidade e exposição solar em participantes do sistema de hipertensão arterial e diabetes melito. Semina: Ciências Biológicas e da Saúde, Londrina, v. 35, n. 2, p. 103-114, jul./dez. 2014.

SARAIVA, Gabriela Luporini et al. Prevalência da deficiência, insuficiência de vitamina $\mathrm{D}$ e hiperparatiroidismo secundário em idosos institucionalizados e moradores na comunidade da cidade de São Paulo, Brasil. Arquivos Brasileiros de Endocrinologia e Metabologia, São Paulo, v. 51, n. 3, p. 437-442, abr. 2007.

SILVA, Barbara Costa Campos et al. Prevalência de deficiência e insuficiência de vitamina D e sua correlação com PTH, marcadores de remodelação óssea e densidade mineral óssea, em pacientes ambulatoriais. Arquivos Brasileiros de Endocrinologia e Metabologia, São Paulo, v. 52, n. 3, p. 482-487, set. 2008

SOARES, Lisandra Delfino de Albuquerque et al. Analysis of motor performance associated with the nutritional status of the elderly enrolled in the Family Health Program in the municipality of Vitória de Santo Antão in the state of Pernambuco. Ciência \& Saúde Coletiva, Rio de Janeiro, v. 17, n. 5, p. 1297-1304, mai. 2012

SOCIEDADE BRASILEIRA DE GERIATRIA E GERONTOLOGIA (SBGG). Quedas em idosos. São Paulo: Projeto Diretrizes: Associação Médica Brasileira e Conselho Federal de Medicina, 2001. Disponível em: <http://www.portalmedico.org.br/diretrizes/ quedas_idosos.pdf>. Acesso em: 20 jan. 2015. 
SOCIEDADEBRASILEIRA DE PATOLOGIA CLÍNICA/MEDICINA LABORATORIAL. ReCOmendações para coleta de sangue venoso. 2. ed. Barueri, SP: Minha Editora, 2010.

TASKAPAN, Hulya et al. Vitamin D and muscle strength, functional ability and balance in peritoneal dialysis patients with vitamin D deficiency. Clinic Nephrology, Rockledge, v. 76, n. 2 , p. 110-116. 2011

VASCONCELOS, Karina Simone de Souza; DIAS, João Marcos Domingues; DIAS, Rosângela Correa. Dificuldades funcionais em mulheres obesas com osteoartrite de joelhos: relação entre percepção subjetiva e desempenho motor. Fisioterapia e Pesquisa, São Paulo, v. 14, n. 3, p. 55-61, set. 2007.

VIRTUOSO, Janeisa Franck et al. Perfil de morbidade referida e padrão de acesso a serviços de saúde por idosos praticantes de atividade física. Ciência e Saúde Coletiva, Rio de Janeiro, v. 17, n. 1, p. 23-31, jan. 2012.

WORLD HEALTH ORGANIZATION (WHO). World Health Statistics 2015. Geneva: WHO, 2015

ZHU, Kun et al. A Randomized Controlled Trial of the Effects of Vitamin D on Muscle Strength and Mobility in Older Women with Vitamin D Insufficiency. The American Geriatrics Society, New York, v. 58, n. 11, p. 2063-2068, nov. 2010.

Data de Submissão: 17/09/2014

Data de Aprovação: 25/03/2018 
